COMMENTARY

\title{
Attitudes Toward People with Epilepsy in Libya, the Conundrum Continues!
}

\author{
Ashraf M Rajab* \\ Department of Medicine, Benghazi Medical Center, Faculty of Medicine, Benghazi University, Libya
}

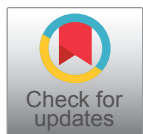

*Corresponding author: Ashraf M Rajab, Department of Medicine, Benghazi Medical Center, Faculty of Medicine, Benghazi University, Benghazi, Libya

A close and contemplative look at the published neurology literature makes it clear to the reader that epilepsy research possesses more popularity and occupies a higher position in the plethora of scholarly medical writings.

Perhaps the reason behind this is that epilepsy nowadays has become more than just transient seizures that are treated with drugs to put an end to seizure recurrence, but rather it is a disease that permeates all aspects of the patient's life.

Epilepsy affects not only the physical aspects of life but it extends to affect psychological, social and professional lives via the worst mechanism of all: Stigmatization.

All of the effects and consequences of epilepsy require scientific attention, and further research efforts.

In the midst of the exponentially growing epilepsy research worldwide, both the scarcity and paucity of studies about Libyan patients with epilepsy (PWE) becomes more apparently evident.

The last study in Benghazi - Libya to determine the prevalence of Epilepsy was conducted in 1986 by Sridharan R, et al. [1] and there are only a few studies that aimed at figuring out the attitudes toward PWE $[2,3]$ however none of them aimed to examine PWE's attitudes toward epilepsy.

Moreover, there is no published data about opinions and perspectives of Libyan PWE with regard to the stigma and how they cope with it? "if they could", what are their levels of knowledge about epilepsy? And what is the extent of negative attitudes practiced toward them?

We admit that epilepsy research in Libya did not provide the complete picture of the situation that can answer these questions so far, as Libyan PWE are still under-represented in the publications.

These unsolved questions and many others represented the impetus for conducting our research [4] in which a sample of Libyan people with and without epilepsy was interviewed and their knowledge about epilepsy and attitudes toward PWE were evaluated and compared to results of similar studies from around the world.

Knowing the Libyan people's attitudes and views toward epilepsy is of immense value to the local health policy makers, as hopefully this will permit further improvement of the Libyans' health literacy planning.

Although many Libyan neurologists are used to critiquing and correcting the widespread number of misconceptions pertaining to epilepsy in their daily practice, unfortunately only a few of these corrections leak out and reach the general public with the vicious cycle of ignorance, misconception and stigma continuing.

The point that distinguished this study was its uniqueness in exploring attitudes of Libyan PWE and others toward epilepsy, although PWE constituted the minority of the participants.

We think that it was surprising to many readers that PWE were on a par with those without epilepsy in 
terms of their attitudes toward epilepsy, as there was no statistically significant difference between the two groups.

It was also worth contemplating and thinking of the finding that $19 \%$ of participants thought that putting a key in PWE's hand will instantly stop the seizure by one way or the other!, a ritual that sounds irrational in the first place, besides that it has not been documented in any previous publication neither locally, regionally nor internationally as far as we know.

It was hard enough to justify and analyze what caused this practice to emerge in the Libyan society; it is difficult to rationalize the emergence of such a practice for which conflicting explanations exist even among those practicing it.

There is no doubt that the inherited misconceptions in this regard are intertwined and sophisticated as they have probably grown worse and worse especially over the past few decades in Libya in which the role of public health education was neglected.

It has been assumed that superstitions about epilepsy and about other diseases abound in environments where formal education and health literacy decreases as lack of education is a breeding ground for misconceptions and it probably lends support to this assumption that the only demographic factor in this study that correlated strongly with positive attitudes toward PWE was the higher educational level.

Through the answers of the participants about the curability of epilepsy and the disease fatality, an optimistic tendency toward epilepsy was perceived even though it is not scientifically accurate, as more than half of the interviewees said that epilepsy cannot lead to death.

Perhaps what happened was a kind of good intentioned bias! A bias in which even the literate participants have fallen in in an attempt to provide what they thought of as the model answer.

The interviewed participants were informed that the study aims to explore knowledge and attitudes toward epilepsy and probably some of them tended to over sympathize to PWE to meet the ideal goals of the interview, maybe extrapolation and analogy have caused them to deny the adverse health consequences of epilepsy and they said epilepsy does not lead to death.

If this was the matter, it is considered a bias but this time in favor of PWE.

While we value positive attitudes toward epilepsy patients, we hope all forms of bias and prejudice are avoided either against or "with" PWE.

It is not by any means in the interest of PWE to deny disease facts and to be blind to its complications, since this would be a dangerous matter that would cause expectedly harmful consequences.

Yes, epilepsy as a cause of death is an issue that belongs to knowledge assessment, but when a participant denies a fact because it sounds derogatory the matter then turns into attitudes.

There are many people who reject some epilepsy facts because they think of these facts as derogatory and this could be named as (the good intentioned non in sighted positive attitudes) if you will, it is an area that needs further studies and solution.

So, what are the positive attitudes we are looking for and recommending to our Libyan society?

The required positive attitude toward PWE is that unbiased, in sighted attitude that recognizes and accepts the scientific facts that include disease manifestations and consequences.

The recommended in sighted positive attitude is a set of qualities that enables PWE to engage in group and social works with a sense of belonging to this community, this in turn will relieve the effects of stigma that burden PWE.

Society's understanding, acceptance and respect are the qualities that the in sighted positive attitude builds upon.

Lastly, we find it necessary to call upon official institutions to take appropriate actions on all levels to reduce stigma and to spread in sighted positive attitudes toward epilepsy, because if actions are not taken the dilemma of negative attitudes and stigma will continue and even increase if neglected as the time goes by.

The article was a sincere attempt to shed light on the current state of Attitudes toward PWE in Libya, and to draw attention toward the need for further research regarding this sensitive issue, and the door for further constructive research is still widely open.

\section{References}

1. Sridharan R, Radhakrishnan K, Ashok PP, Mousa ME (1986) Epidemiological and clinical study of epilepsy in Benghazi, Libya. Epilepsia 27: 60-65.

2. Taher YA, Al-Gomati MA, Samud AM, El-Taher FE (2018) Knowledge and attitudes toward epilepsy among Libyan parents resident in Tripoli. Lib J Med Sci 2: 102-107.

3. Alhagamhmad MH, Shembesh NM (2018) Investigating the awareness, behavior, and attitude toward epilepsy among university students in Benghazi, Libya. Epilepsy Behav 83: 22-27.

4. Rajab AM, Lawgaly SA, El Beshari M, Bennour AM (2021) Knowledge and attitudes toward epilepsy among Libyan people with and without epilepsy in a tertiary medical center. Epilepsy Behav 121: 108023.
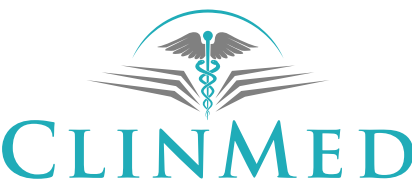

INTERNATIONAL LIBRARY 